International Mathematical Forum, Vol. 8, 2013, no. 22, 1049 - 1056

HIKARI Ltd, www.m-hikari.com

http://dx.doi.org/10.12988/imf.2013.3465

\title{
On Euler's Synthetic Demonstration of Pappus' Construction of an Ellipse from a Pair of Conjugate Diameters
}

\author{
Brian J. Mcartin \\ Applied Mathematics, Kettering University \\ 1700 University Avenue, Flint, MI 48504-6214 USA \\ bmccarti@kettering.edu
}

Copyright (C) 2013 Brian J. M들. Cartin. This is an open access article distributed under the Creative Commons Attribution License, which permits unrestricted use, distribution, and reproduction in any medium, provided the original work is properly cited.

\begin{abstract}
This expository paper reviews Pappus' construction of an ellipse from a pair of conjugate diameters [3] together with Euler's synthetic demonstration [2] of $1750(3)$.
\end{abstract}

Mathematics Subject Classification: 00-01,01-01, 51M15, 51N20

Keywords: conic section, ellipse, conjugate diameter, Pappus, Euler

\section{Introduction}

In Book VIII of his Collection ( $4^{\text {th }}$ Century A.D.), Pappus was the first to provide an explicit construction of the principal axes of an ellipse from a given pair of its conjugate diameters (see Figure 1) [3, pp. 437-438]. (Consult [1] for the relevant history of the conic sections, [9] for their synthetic treatment and [8] for their corresponding analytic treatment.) However, he omitted any demonstration of the validity of his construction. Thus, it was left for Euler $(1750(3))[2]$ to provide the first synthetic demonstration of Pappus' construction.

Unfortunately for the modern reader, Euler's original work on this topic is available only in Latin and presumes a familiarity with properties of the conic 
sections which is no longer commonplace. (This latter difficulty also awaits modern readers of Newton's Principia [7].) It is true that Euler's demonstration of Pappus' construction is discussed by Salmon [8, p. 173] but the treatment therein (where, incidentally, reference is made to neither Pappus nor Euler) is so condensed as to be nearly indigestible.

Thus, it is the express purpose of the present note to provide a selfcontained and reconstituted exposition of Euler's demonstration of Pappus' construction. A concise specification of Pappus' construction is followed by a detailed description of Euler's demonstration with some background geometric propositions relegated to an appendix.

\section{Pappus' Construction [3, pp. 437-438]}

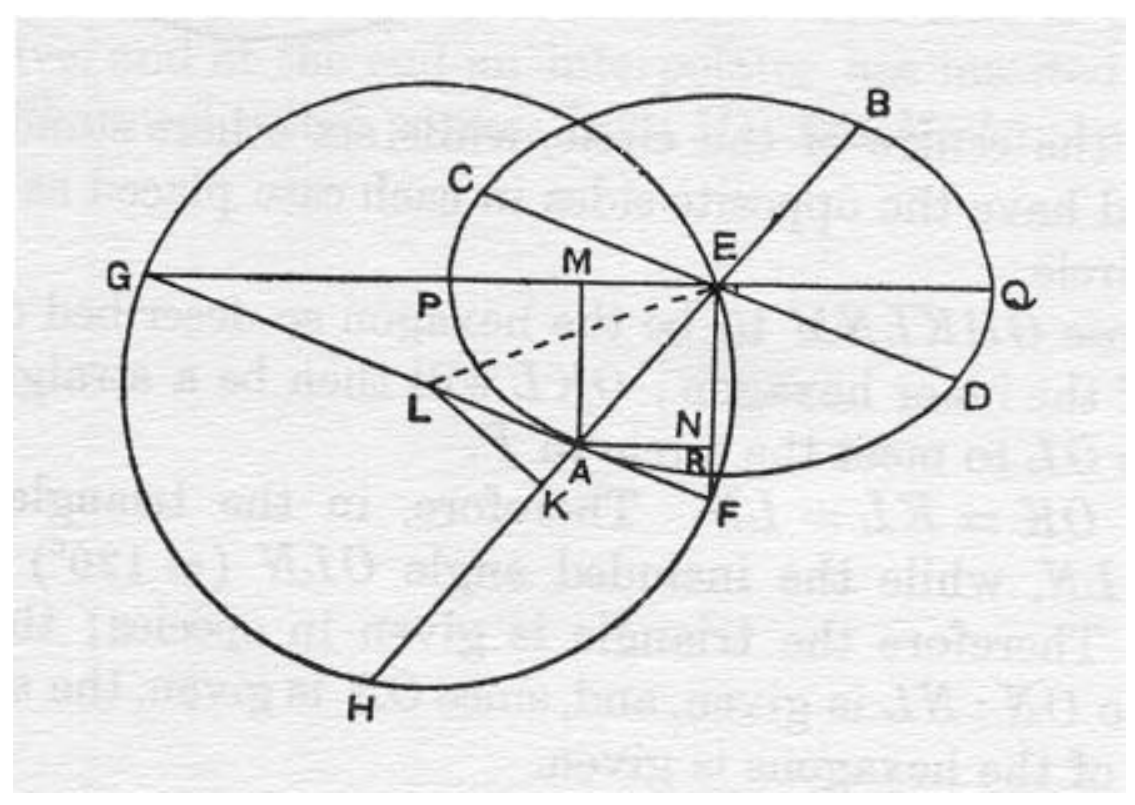

Figure 1: Pappus' Construction

At the conclusion of Chapter 17 of Book VII of The Collection [3, pp. 437-438], Pappus presents

Proposition 1 (Propostion 14: Pappus' Construction) Problem: Given two conjugate diameters of an ellipse, to find the principal axes in both position and magnitude.

- Given (see Figure 1): Conjugate diameters $A B$ \& $C D$ intersecting at center $E$. 
- Step 1: Produce EA to $H$ so that $E A \cdot A H=D E^{2}$.

- Step 2: Draw a line, $\mathcal{L}$, through $A \| C D$.

- Step 3: Bisect EH at K.

- Step 4: Draw $K L \perp E H$ meeting $\mathcal{L}$ at $L$.

- Step 5: With $L$ as center and LE as radius, draw a circle cutting $\mathcal{L}$ at $F$ and $G$.

- Step 6a: Join EF, then draw AM $\| E F$ with $M$ lying on EG.

- Step 6b: Join EG, then draw $A N \| E G$ with $N$ lying on $E F$.

- Step 7a: Construct $P$ on $E G$ such that $E P^{2}=G E \cdot E M$.

- Step 7b: Construct $R$ on $E F$ such that $E R^{2}=F E \cdot E N$.

- Step 8: EP and ER are the principal semiaxes.

Pappus omits any demonstration of the validity of this construction.

\section{Euler's Demonstration [2, pp. 231-232]}

Demonstration 1 (Euler's Demonstration of Pappus' Construction) With reference to Figure 2/Fig. 5, let $C E$ and $C F$ be the given conjugate semidiameters of the ellipse.

- At F, construct a line parallel to $C E$ which will be tangent to the ellipse at $F$ as $C E$ and $C F$ are conjugate semidiameters.

- The principal axes will intercept this tangent line at the presently unknown points $G$ and $H$ so that $C G$ and $C H$ will fall along the principal axes of the ellipse and $\angle G C H$ will be a right angle.

- Since $\angle G C H$ is a right angle, the circle passing through $G, C$ and $H$ will have $G H$ as a diameter (by Euclid III.31) so that its center is at the likewise presently unknown midpoint I of $G H$.

- By Smith III.XVI/Corollary, FG $F H=C E^{2}$.

- The presently unknown circle will also pass through the line $C F$ produced to $K$ and, by Euclid III.35, CF $\cdot F K=F G \cdot F H$. 
- Combining these last two equations, we conclude that $C F \cdot F K=C E^{2}$, i. e. $F K$ is the third proportional to $C F$ and $C E$ which are given. Thus, the point $K$ can be constructed.

- Now that $K$ is available to us, we may bisect $C K$ at $L$ and erect the normal LI cutting $G H$ at the now determined center $I$.

- Once the center $I$ is available, we may use either $I C$ or $I K$ as radius to construct the circle cutting the tangent line at $G$ and $H$ which are now finally available.

- Since we know that the principal axes lie along $C G$ and $C H$, all we need now do is to determine their lengths. This can be accomplished with the aid of Smith III.III/IV. First drop a perpendicular from $F$ onto $C G / C H$ and call the foot of the perpendicular $M / N$. Then, the required length of a semiaxis is given by the mean proportional of $C M \& C G / C N \& C H$.

Q.E.F.

\section{Conclusion}

As is readily apparent from the preceding, the synthetic approach to this problem does not readily permit the distinguishing of the major and minor axes of the ellipse. This is important in applications such as orthogonal linear regression where the major axis lies along the line of best fit while the minor axis lies along the line of worst fit [5]. This difficulty is remedied by the vector analytic approach to this problem described in [6].

\section{A Geometric Propositions}

Proposition 2 (Euclid III.1 [4]) In a circle the angle in the semicircle is right, that in a greater segment less than a right angle, and that in a less segment greater than a right angle; and further the angle of the greater segment is greater than a right angle, and the angle of the less segment less than a right angle. (In particular, $\angle B A C$ in Figure 3 is a right angle.)

Proposition 3 (Euclid III.31 [4]) The center of a circle lies on the perpendicular bisector of any chord of the circle. (In Figure 4, the center $F$ of circle $A B C$ lies on the perpendicular bisector DE of chord $A B$.) 
Proposition 4 (Euclid III.35 [4]) If in a circle two straight lines cut one another, the rectangle contained by the segments of the one is equal to the rectangle contained by the segments of the other. (In Figure 5, AE $E C=$ $D E \cdot E B$.

Proposition 5 (Smith III.III/IV [9]) If the tangent at any point $P$ of an ellipse meets the major/minor axis $C A / C B$ produced in $T / t$, and $P N / P n$ be the perpendicular to the axis; then will $C N \cdot C T=C A^{2} / C n \cdot C t=C B^{2}$. (See Figure 6.)

Proposition 6 (Smith III.XVI/Corollary [9]) If the tangent at $P$ to an ellipse be cut in $T, T^{\prime}$ by any pair of conjugate diameters, and $C D$ be the semidiameter conjugate to $C P$; then will $T P \cdot P T^{\prime}=C D^{2}$. (See Figure \%.)

\section{References}

[1] J. L. Coolidge, History of the Conic Sections and Quadric Surfaces, Dover, New York, NY, 1968(1945).

[2] L. Eulero, Solutio Problematis Geometrici, Novi Commentarii Academiae Scientiarum Petropolitanae, Tom. III (1750/1751), 1753, pp. 224-234+1 diagram (Tab. IV), http://eulerarchive.maa.org/pages/E192.html.

[3] T. L. Heath, A History of Greek Mathematics, Volume II: From Aristarchus to Diophantus, Dover, New York, NY, 1981(1921).

[4] T. L. Heath, The Thirteen Books of Euclid's Elements, Volume 2: Books III-IX, Second Edition Unabridged, Dover, New York, NY, 1956.

[5] B. J. McCartin, A Geometric Characterization of Linear Regression, Statistics, Vol. 37, No. 2, 2003, pp. 101-117.

[6] B. J. McCartin, A Vector Analytic Demonstration of Pappus' Construction of an Ellipse from a Pair of Conjugate Diameters, Applied Mathematical Sciences, Vol. 8, 2014 (to appear).

[7] I. Newton, Principia, Second Edition, University of California Press, Berkeley, CA, 1971(1713).

[8] G. Salmon, A Treatise on Conic Sections, Sixth Edition, Chelsea, New York, NY, 1954(1879).

[9] C. Smith, Geometrical Conics, Macmillan, London, 1907.

Received: April 1, 2013 


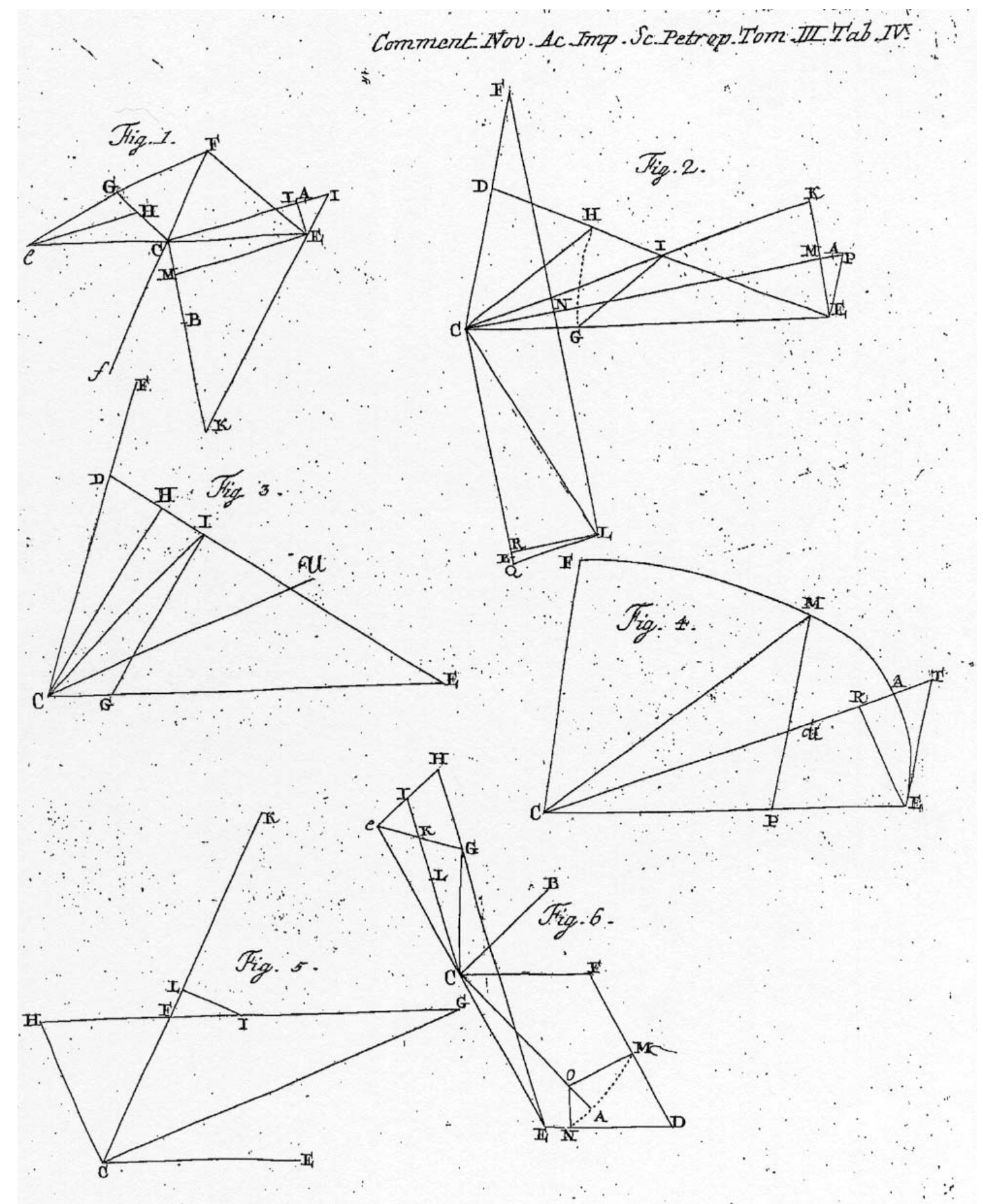

Figure 2: Euler's Demonstration [2] 


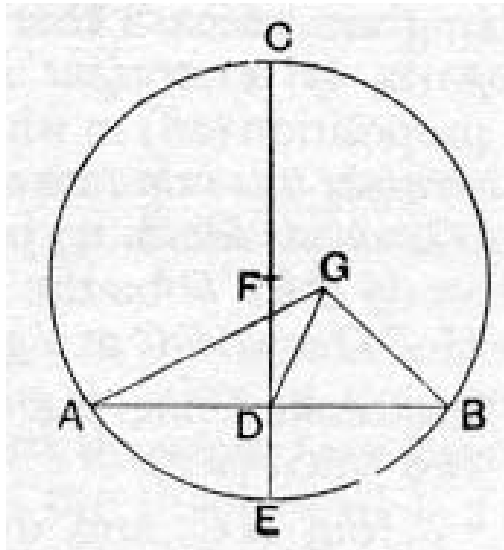

Figure 3: Euclid Proposition III.1 [4, pp. 6-8]

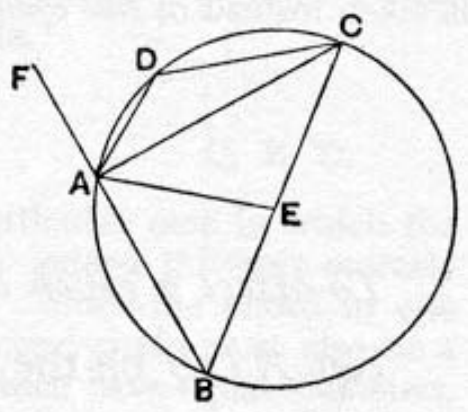

Figure 4: Euclid Proposition III.31 [4, pp. 61-62]

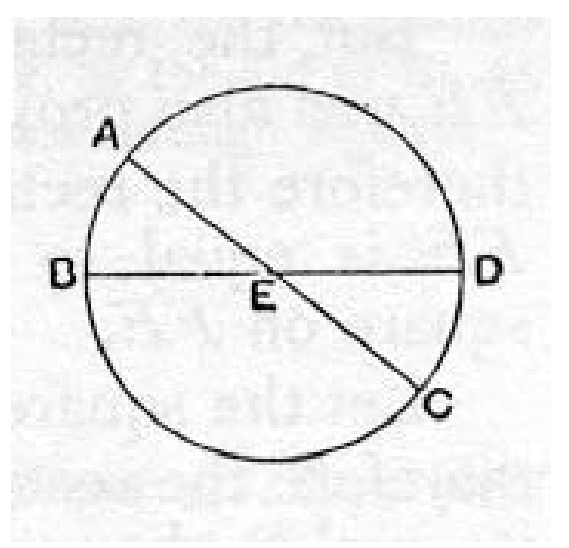

Figure 5: Euclid Proposition III.35 [4, pp. 71-73] 


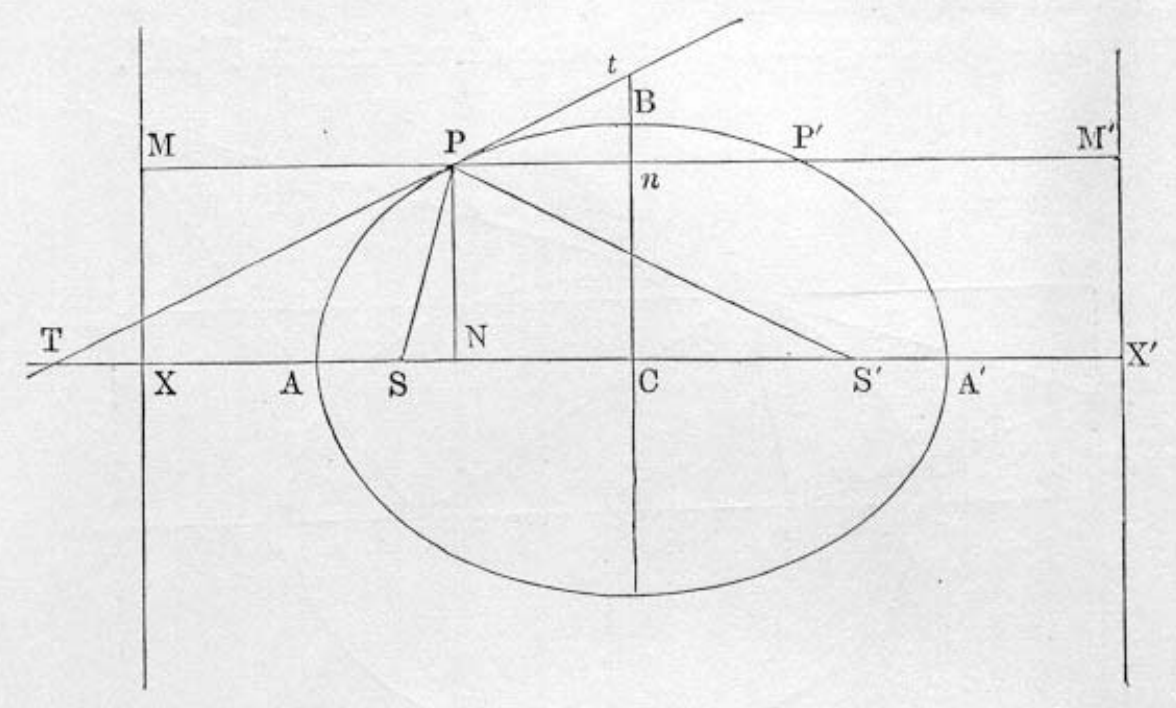

Figure 6: Smith Proposition III.III/IV [9, p. 86]

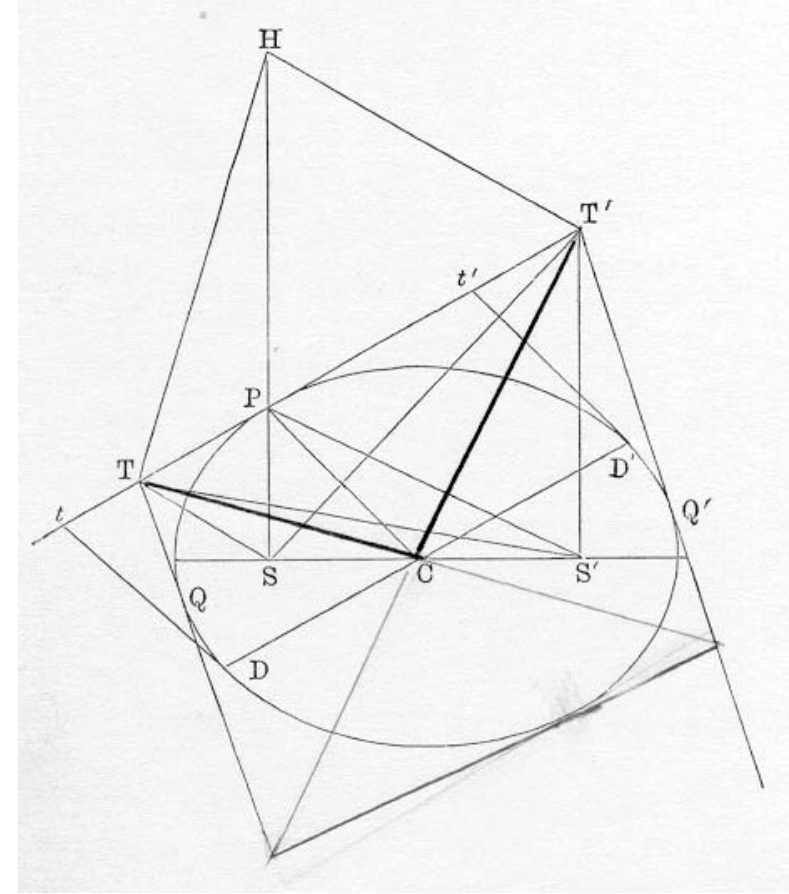

Figure 7: Smith Proposition III.XVI/Corollary [9, pp. 102-103] 